\title{
Acute Paraparesis after Epidural Corticosteroid Injection Revealing Spinal Dural Arteriovenous Fistula in a HIV Patient
}

\author{
Anouk Le Goueff ${ }^{1}$, Nicolas Mavroudakis², Benjamin Mine ${ }^{3}$, Olivier De Witte ${ }^{4}$, Gauthier Remiche ${ }^{2}$ \\ ${ }^{1}$ Internal Medicine Department, Hôpital Erasme, Université Libre de Bruxelles, Brussels, Belgium \\ ${ }^{2}$ Centre de Référence Neuromusculaire, Service de Neurologie, Hôpital Erasme, Université Libre de Bruxelles, Brussels, Belgium \\ ${ }^{3}$ Interventional Neuroradiology Department, Hôpital Erasme, Université Libre de Bruxelles, Brussels, Belgium \\ ${ }^{4}$ Neurosurgery Department, Hôpital Erasme, Université Libre de Bruxelles, Brussels, Belgium
}

\section{Doi: 10.12890/2020_001673 - European Journal of Case Reports in Internal Medicine - ๔ EFIM 2020}

Received:17/04/2020

Accepted: $23 / 04 / 2020$

Published: 08/07/2020

How to cite this article: Le Goueff A, Mavroudakis N, Mine B, De Witte O, Remiche G. Acute paraparesis after epidural corticosteroid injection revealing spinal dural arteriovenous fistula in a HIV patient. EJCRIM 2020;7: doi:10.12890/2020_001673.

Conflicts of Interests: From 2008 to 2017, Dr Remiche received several financial awards from Sanofi Genzyme, Santhera Pharmaceuticals, CSL Behring and C.A.F.-D.C.F. cvba-scrl such as: travel grants and financial support in order to attend scientific meetings, a consultancy honorarium, a speaker honorarium, a grant to conduct academic study and a grant to organize a scientific meeting. No potential conflict of interest is reported by the other authors.

This article is licensed under a Commons Attribution Non-Commercial 4.0 License

\section{ABSTRACT}

Spinal dural arteriovenous fistulas (SDAVFs) are often misdiagnosed as their symptoms are non-specific, leading to treatment delay and a poor outcome.

We describe the case of a 53-year-old man with a history of progressive paraparesis that worsened abruptly after an epidural corticosteroid injection. We highlight here the need for high diagnostic suspicion for an SDAVF in patients deteriorating after an epidural injection and an indication of repeated spine imaging in such cases.

Finally, this is the first reported case of an SDAVF in a HIV-positive patient and it emphasizes the need for a broad differential diagnosis.

\section{LEARNING POINTS}

- Consider an SDAVF in cases of slowly progressive paraparesis and hypoaesthesia, especially if symptoms worsen after an epidural injection.

- The need for an in-depth work-up including repeated angiographic explorations in cases where no malformation is found in a straightforward manner.

- In a patient infected with HIV, even though a broad range of neuromuscular diseases can be suspected, non-related aetiologies should not be forgotten.

\section{KEYWORDS}

Arteriovenous fistula, paraplegia, spinal cord, epidural analgesia

\section{INTRODUCTION}

A spinal dural arteriovenous fistula (SDAVF) is an arteriovenous shunt, most frequently localized in the lumbar spine, that leads to congestive myelopathy. This acquired lesion is rare, with an incidence of 5-10 cases per million per year, yet it represents the most frequent spinal vascular malformation. Its clinical presentation is non-specific, initially presenting with radicular pain in lower limbs, paraesthesia and gait disturbances, thus leading to misdiagnosis that delays treatment and increases long-term disability ${ }^{[1,2]}$. 


\section{CASE DESCRIPTION}

We report the case of a 53-year-old male who developed an acute cauda equina syndrome within hours of an analgesic epidural injection. His past medical history consisted of well-controlled HIV infection and Buerger disease. His daily treatments were etravirine, darunavir, ritonavir, raltegravir, allopurinol, colestyramine, zopiclone, carvedilol and bromazepam.

He presented initially with cramps, paresis and hypoaesthesia of his toes that slowly progressed proximally. After 7 months of evolution, he reported hypoaesthesia and paraparesis of his entire lower limbs that limited his walking perimeter to a few metres aided by 2 crutches. No urogenital dysfunction was reported at that time. During that period, physical examination demonstrated ataxic gait, lower limb hypopallaesthesia, hyporeflexia, absent Babinski sign and paresis at 4/5 with respect to the Medical Research Council (MRC) score. Electromyography (EMG) showed severe sensory-motor axonal polyneuropathy. In order to rule out lumbar compression, a lumbar MRI was performed and objectified an L5-S1 right herniated disc. Given the worsening of symptomatology and severe lower back pain, the patient eventually received an epidural injection of $80 \mathrm{mg}$ methylprednisolone acetate into the L4-L5 medullar epidural space that worsened the paraparesis within hours, with a 3/5 MRC score reached for the lower limbs. Moreover, a Th10 sensory hypoaesthesia level and urinary retention were observed. Extensive blood and cerebrospinal fluid work-up was unremarkable for infectious or autoimmune aetiologies.

Methods and Procedures

A whole spine MRI showed Th9-L1 intramedullary hypersignal while magnetic resonance angiography (MRA) confirmed these findings and revealed serpiginous vascular structures posterior to the spinal cord at the Th10-Th11 level (Fig. 1). Given the high clinical and radiological suspicion for an SDAVF, 2 spinal angiographies were performed and individualized Th5-L3 pedicles, but showed no fistula. Finally, a third medullar arteriogram was performed and highlighted a left Th4 perimedullary fistula (Fig. 2). After surgical electrocoagulation of the shunting zone, the patient regained autonomy regarding all daily living activities. However, 1 month later, his strength deteriorated and an additional angiograph revealed a new post-surgical arteriovenous fistula.

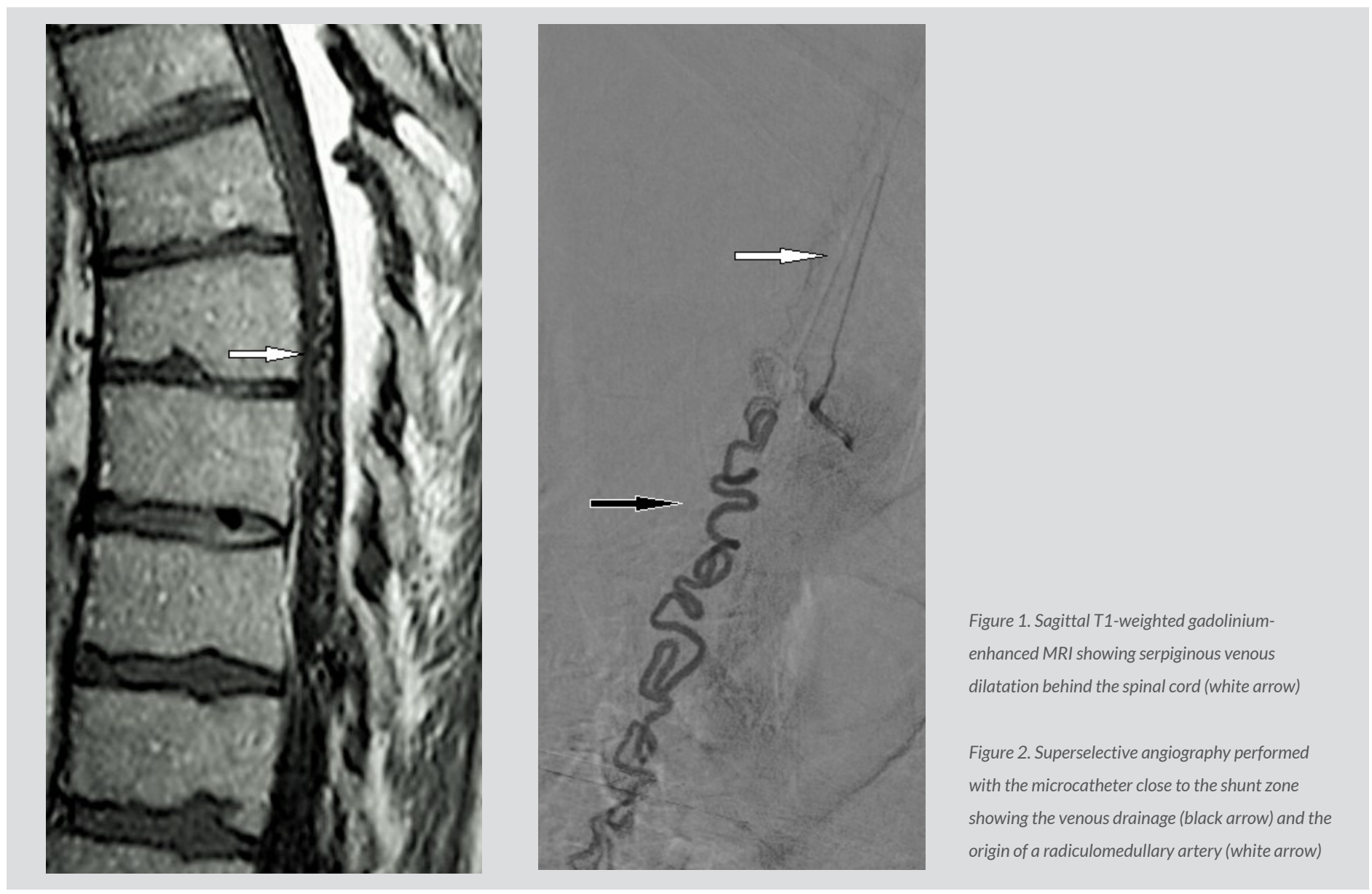




\section{DISCUSSION}

An SDAVF is an arteriovenous shunt, most frequently localized in the lumbar spine, which is fed by a dural artery and drained by the perimedullary venous system. It mostly presents as slowly ascending paraparesis and sensory impairment, eventually evolving to paraplegia, neuropathic pain and bladder, bowel and sexual dysfunction if diagnosis and treatment do not take place during the initial stages. However, the fistula often remains undiagnosed as initial symptoms are non-specific and can mimic other diseases such as polyneuropathy, herniated disc, multiple sclerosis or tumours. Diagnostic delay retards efficient treatment and consequently alters long-term functional prognosis ${ }^{[1,2]}$. Epidural corticosteroid injection is well described as a potential acute aggravating factor with respect to SDAVF symptomatology. Therefore, abrupt clinical worsening after such procedures should raise high clinical suspicion for this diagnosis. Moreover, it is recommended that this condition be excluded prior to any epidural injection ${ }^{[3,4]}$.

MRI investigations in the context of SDAVFs typically show centro-medullar and multisegmental cord hypersignal in T2-weighted sequences that does not necessarily correspond to the level of the fistula. Consequently, extended MRI should be prioritized over segmental MRI only for SDAVF assessment, in order to avoid false negative results ${ }^{[1,2]}$.

Once this diagnosis is suspected, further imaging by MRA is recommended in order to localize the fistula level. Finally, conventional angiography remains the gold-standard procedure to confirm the diagnosis and to proceed with selective injection and occlusion of the feeder arteries that are the core measures for treatment ${ }^{[1,2]}$. However, its sensitivity is not absolute and when clinical suspicion is high, conventional angiography should be repeated, as illustrated here.

Initial treatment success rates are as high as $98 \%$ and up to two-thirds of patients can improve their motor symptoms ${ }^{[1-4]}$. However, some patients will experience clinical worsening within a few weeks of recovery, secondary to a recanalization of the fistula or to new shunt formation. Therefore, surveillance imaging is recommended on a regular basis, or at least, when worsening of the symptoms occurs ${ }^{[1,2]}$.

HIV has been associated with a broad range of neuromuscular diseases. In a patient presenting with proximally evolving paraesthesia, hypoaesthesia and paresis, the clinician should consider distal symmetrical polyneuropathy and chronic inflammatory demyelinating polyradiculoneuropathy (CIDP). However, unlike in our case, both entities usually affect patients with poorly controlled HIV, or those starting treatment with old antiretroviral agents (for example, zalcitabine, stavudine and didanosine) ${ }^{[5]}$. To the best of our knowledge, this is the first reported case of an SDAVF in a HIV-positive patient. This is most probably an incidental finding.

\section{REFERENCES}

1. Krings T, Geibprasert S. Spinal dural arteriovenous fistulas. Am J Neuroradiol 2009;30(1):639-647.

2. Lenck S, Bernat A, Bresson D, Labeyrie M, Saint-Maurice J, Froelich S, et al. Spinal dural arteriovenous fistula. J Spine 2016;5(1):1-4.

3. Oliver T, Sorensen M, Arthur A. Endovascular treatment for acute paraplegia after epidural steroid injection in a patient with spinal dural arteriovenous malformation. $J$ Neurosurg 2012;17(1):251-255.

4. Hetts S, Narvid J, Singh T, Meagher S, Corcoran K, Higashida R, et al. Association between lumbar epidural injection and development of acute paraparesis in patients with spinal dural arteriovenous fistulas. Am J Neuroradiol 2007;28(1):581-583.

5. Prior DE, Song N, Cohen JA. Neuromuscular diseases associated with Human Immunodeficiency Virus infection. J Neurol Sci 2018;387:27-36. 